\title{
Impact of System Quality on Users' Satisfaction in Continuation of the Use of e-Learning System
}

\author{
Abdulhakim Elmoawe Dreheeb*, Nurlida Basir, Norasikin Fabil \\ Fakulti Sains dan Teknologi, Universiti Sains Islam Malaysia (USIM), 71800 Nilai, Negeri Sembilan, Malaysia. \\ * Corresponding author. Email: asid1433@yahoo.com \\ Manuscript submitted October 16, 2015; accepted December 15, 2015. \\ doi: 10.17706/ijeeee.2016.6.1.13-20
}

\begin{abstract}
The e-learning is a future education that would substitute to the traditional learning, which overcomes the spatial and temporal limitations. The e-learning field growth rapidly in the recently years due to its importance in the private and public sectors. This paper focuses on the system quality of e-learning. The e-learning system depends on the quality to be successful and the real success is sustained usage. The users of e-learning system will stop using such system if the quality is poor, where often the users reject the system unless they try it, where the intentions of continuing using the system are still weak. There are several attributes and functionalities that can have an impact on the use of e-learning based on user perspective, such as are usability, reliability and efficiency. These quality attributes are used to reflect the quality of the software product. The intended objective of this study is to develop an appropriate model for e-learning to satisfy the users from the side of using the e-learning system, where carried the discussion of twenty-four model with thirty attributes. Finally, the result of this study adopted the process of structural equation model which indicated that the hypotheses have positive relations.
\end{abstract}

Key words: E-learning systems, system quality factors, satisfaction, continue to use.

\section{Introduction}

The success of any software depends on the quality which contributes as main pillars that should be taken in consideration, where the quality is an important issue for the development of the whole kinds of software, specifically in education where the software is used as mean for e-learning [1]. The first essential step to attaining e-learning success is the initial acceptance from the end users, yet the real success is sustained usage. Importantly, there are several attributes affecting the users' satisfaction to continue using e-learning, these attributes are important to assist the developers to design an ideal contents [2]. The main attributes of fulfilling the former are categorized under the System Quality as the usability, reliability and efficiency where they have been found as top three attributes in many models. However, e-learning provides a modern way for the possibility of learning to enhance the knowledge and skills through the internet and computer networks by shifting from teaching and memorization to learning and thinking. Moreover, e-learning is the use of technology and information networks to perform the process of learning [3]. Thus, in general, neglecting the system quality probably leads to the dropout rates of e-learning where percent between 20 to 80 has been reported, so decreasing the dropout rate is one of the major challenges of employing e-learning systems [4].

\section{Background}


There are many software quality models that drawing the foundation for developing an appropriate model for e-learning. A quality model is defined as set of attributes and the correlated relationship between them, which provide the basis for specifying the quality requirements and evaluating the product quality [5]. These reachable quality models are defined as twenty-four models include but are not limited to McCall, Boehm, FURPS, Dromey, QMOOD, ISO 9216 and etc.

Table 1. Comparison between Quality Models with Their Attributes

\begin{tabular}{|c|c|c|c|c|c|c|c|c|c|c|c|c|c|c|c|c|c|c|c|c|c|c|c|c|c|c|}
\hline 之 & $\frac{\bar{g}}{\frac{0}{0}}$ & $\frac{n}{2}$ & 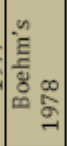 & 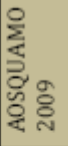 & 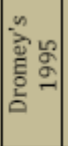 & 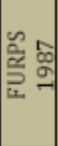 & 정 & 응 & 突 & 을 & 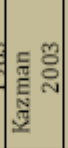 & 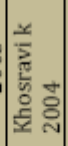 & 똔 & 或 & 종 & 桨 & 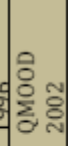 & ș & 용요 & 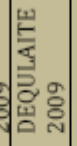 & 롱연 & 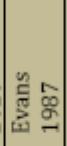 & 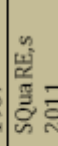 & 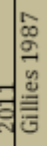 & 영 & 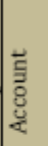 \\
\hline 1 & Correctness & $\checkmark$ & & & & & $\checkmark$ & & $\checkmark$ & $\checkmark$ & & & & $\checkmark$ & & & & & & & & $\checkmark$ & & $\checkmark$ & & 7 \\
\hline 2 & Efficiency & $\checkmark$ & $\checkmark$ & $\checkmark$ & $\checkmark$ & & $\checkmark$ & $\checkmark$ & $\checkmark$ & $\checkmark$ & $\checkmark$ & & $\checkmark$ & $\checkmark$ & & $\checkmark$ & & $\checkmark$ & $\checkmark$ & & $\checkmark$ & $\checkmark$ & $\checkmark$ & $\checkmark$ & 8 & 19 \\
\hline 3 & Expandability & & & & & & & & $\checkmark$ & & & & & $\checkmark$ & & & $\checkmark$ & & & $\checkmark$ & & $\checkmark$ & & & & 5 \\
\hline 4 & Flexibility & $\checkmark$ & & & & & $\checkmark$ & & $\checkmark$ & $\checkmark$ & $\checkmark$ & $\checkmark$ & & $\checkmark$ & $\checkmark$ & & $\checkmark$ & & & & & $\checkmark$ & & $\checkmark$ & & 11 \\
\hline 5 & Functionality & & & $\checkmark$ & $\checkmark$ & $\checkmark$ & & $\checkmark$ & & & $\checkmark$ & & $\checkmark$ & & & $\checkmark$ & $\checkmark$ & $\checkmark$ & $\checkmark$ & $\checkmark$ & $\checkmark$ & & $\checkmark$ & & 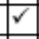 & 14 \\
\hline 6 & Human En & & $\checkmark$ & & & & & & & & & & & & & & & & & & & & & 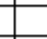 & & 1 \\
\hline 7 & Integrity & $\checkmark$ & & & & & $\checkmark$ & & $\checkmark$ & $\checkmark$ & & & & $\checkmark$ & $\checkmark$ & & & & & & $\checkmark$ & $\checkmark$ & & $\checkmark$ & & 9 \\
\hline 8 & Interoperability & $\checkmark$ & & & & & $\checkmark$ & & $\checkmark$ & $\checkmark$ & & & & $\checkmark$ & & & & & & & $\checkmark$ & $\checkmark$ & & $\checkmark$ & & 8 \\
\hline 9 & Intraoperability & & & & & & & & & $\checkmark$ & & & & & & & & & & & & & & 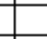 & & 1 \\
\hline 10 & Maintainability & $\checkmark$ & & $\checkmark$ & $\checkmark$ & & $\checkmark$ & $\checkmark$ & $\checkmark$ & $\checkmark$ & $\checkmark$ & & $\checkmark$ & $\checkmark$ & $\checkmark$ & $\checkmark$ & & $\checkmark$ & $\checkmark$ & & & $\checkmark$ & $\checkmark$ & $\checkmark$ & $\checkmark$ & 18 \\
\hline 11 & Modifiability & & $\checkmark$ & & & & & & & & & & & & & & $\checkmark$ & & & $\checkmark$ & $\checkmark$ & & & 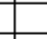 & & 4 \\
\hline 12 & Performance & & & & & $\checkmark$ & & & & & & & & & & & & & & & & & & & & 1 \\
\hline 13 & Portability & $\checkmark$ & $\checkmark$ & $\checkmark$ & $\checkmark$ & & $\checkmark$ & $\checkmark$ & $\checkmark$ & $\checkmark$ & & & $\checkmark$ & $\checkmark$ & $\checkmark$ & $\checkmark$ & & $\checkmark$ & $\checkmark$ & & $\checkmark$ & $\checkmark$ & $\checkmark$ & $\checkmark$ & $r$ & 19 \\
\hline 14 & Supportability & & & & & $\checkmark$ & & & & & & & & & & $\checkmark$ & & & & & & & & 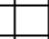 & & 2 \\
\hline 15 & Survivability & & & & & & & & $\checkmark$ & & & & & $\checkmark$ & & & & & & & & & & 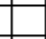 & & 2 \\
\hline 16 & Testability & $\checkmark$ & $\checkmark$ & & & & $\checkmark$ & & & $\checkmark$ & & & & & & & & & & $\checkmark$ & $\checkmark$ & $\checkmark$ & $\checkmark$ & $\checkmark$ & & 10 \\
\hline 17 & Understandability & & $\checkmark$ & & & & & & & & & $\checkmark$ & & & & & $\checkmark$ & & & $\checkmark$ & $\checkmark$ & & $\checkmark$ & & & 6 \\
\hline 18 & Usability & $\checkmark$ & & $\checkmark$ & $\checkmark$ & $\checkmark$ & $\checkmark$ & $\checkmark$ & $\checkmark$ & $\checkmark$ & $\checkmark$ & $\checkmark$ & $\checkmark$ & $\checkmark$ & $\checkmark$ & $\checkmark$ & $\checkmark$ & $\checkmark$ & $\checkmark$ & $\checkmark$ & $\checkmark$ & $\checkmark$ & $\checkmark$ & $\checkmark$ & $\checkmark$ & 23 \\
\hline 19 & Reliability & $\checkmark$ & $\checkmark$ & $\checkmark$ & $\checkmark$ & $\checkmark$ & $\checkmark$ & $\checkmark$ & $\checkmark$ & $\checkmark$ & $\checkmark$ & & $\checkmark$ & $\checkmark$ & $\checkmark$ & $\checkmark$ & & $\checkmark$ & $\checkmark$ & & $\checkmark$ & $\checkmark$ & $\checkmark$ & $\checkmark$ & $\checkmark$ & 21 \\
\hline 20 & Accuracy & & & & & & & & & & & & & & $\checkmark$ & & & & & & $\checkmark$ & & & & & 2 \\
\hline 21 & Verifiability & & & & & & & & $\checkmark$ & & & & & $\checkmark$ & & & & & & & & $\checkmark$ & & & & 3 \\
\hline 22 & Safety & & & & & & & & & & & & & $\checkmark$ & & & & & & & & & & & & 1 \\
\hline 23 & Reusability & $\checkmark$ & & & $\checkmark$ & & $\checkmark$ & & $\checkmark$ & $\checkmark$ & & $\checkmark$ & & $\checkmark$ & $\checkmark$ & & $\checkmark$ & & & $\checkmark$ & & $\checkmark$ & & $\checkmark$ & & 12 \\
\hline 24 & Manageability & & & & & & & & & & & & & $\checkmark$ & & & & & & & & & & & & 1 \\
\hline 25 & Feasibility & & & & & & & & & & & & & & & $\checkmark$ & & & & & & & & & & 1 \\
\hline 26 & Robustness & & & & & & & & & & & $\checkmark$ & & & & & & & & $\checkmark$ & & & & & & 2 \\
\hline 27 & Scalability & & & & & & & & & & & $\checkmark$ & & & & & & & & $\checkmark$ & & & & & & 2 \\
\hline 28 & Modularity & & & & & & & & & & & $\checkmark$ & & & & & $\checkmark$ & & & $\checkmark$ & & & & & & 3 \\
\hline
\end{tabular}

The investigation of these quality models includes the comparison of the available quality attributes which are considered as attributes for system quality. Thirty common attributes have been found in twenty-four quality models which are presented in Table 1 . The common attributes that found in the majority of these models are identified as usability where the frequency was 23 , reliability was 21 , and efficiency was 19 Straight. These attributes were mainly categorized for the system quality from the prospective of the end user, which they play a very important role in building user satisfaction to continue using the e-learning system.

Throughout the investigation of the reachable quality models that available on the database of the libraries which were twenty-four models, the first investigated model was proposed in 1977 by McCall and last investigated model in this study was proposed by Kumar in 2012 which named as aspect-oriented software quality (AOSQ). This study was intended to collect as many as can of the existed quality models to verify the attributes in each of these models. Based on that, it becomes very clear the system quality attributes were very significant in the area of e-learning success. Accurately, these attributes in this study were heavily focused on the prospective of the end-users, which are summarized and detailed in table 1, that initiated from the highest presence in the quality models which they were as follow: usability, reliability and efficiency, where all of these attributes play an important role in obtaining user satisfaction 
to continue using the e-learning system.

\section{Proposed Framework}

The main objective of the proposed framework structure as shown in Fig. 1 is to find out the attributes that affect the users' satisfaction in their use of e-learning, and as the attributes have been centered on the quality of the system, there was a necessity to proof the relationship between the system quality and the satisfaction as well as the relationship between satisfaction and continue to use in order to support the process of developing the final version of the framework, as explained in details below. Therefore, it was found that several models support the relationship positively between system quality and satisfaction such as Model and Continuous Intention to Use E-learning Systems Model. Also, the models that support the positive relationship between satisfactions and continue to use, such as a Personal Response System (PRS), and Expectancy Confirmation Theory (ECT). Based on what stated in the former, it is heavily considered as the motivation to do the same in terms of connecting system quality with satisfaction as well as satisfaction with continue to use.

\subsection{System Quality}

System quality plays an important role in the success of an overall software system. It is considered as a very important aspect for developers, users, and project managers. System quality is the extent to which an industry define a set of desirable features that should be incorporated into the product in order to enhance its lifetime performance [6]. Moreover, according to the Information System Model, system quality is a critical success attributes that influences user satisfaction and intention to use [7].

\subsubsection{Usability}

Usability is one of the most important attributes for all software quality models. It is one of the crucial attributes in the development of successful collaborative software applications [8]. Usability plays a significant role towards the success of e-learning applications [9]. Moreover, usability is the basic parameter for the evaluation of e-learning technologies and systems. It is the capability of the software product to be understood learned, used and be attractive to the user when used under specified conditions.

\subsubsection{Reliability}

Reliability is considered as one of the most important attributes of software quality [10]. The reliability is a set of attributes that relate to the capability of the software to maintain its level of performance under stated conditions for a stated period of time. The system needs to maintain a listed level of performance in case of software faults with minimum crashes. The system also must have the ability to reestablish its level of performance and continue to produce the same results [11].

\subsubsection{Efficiency}

Efficiency is one of the attributes that was accounted very important attributes of software quality. Efficiency is the capability of the software product to provide appropriate performance, relative to the amount of resources used under stated conditions. Users expect the system to run in an efficient manner when utilizing an e-learning environment. System response - time performance, as well as page and graphics generation speed, must be high enough to satisfy learners' needs. [11].

\subsection{Satisfaction}

User satisfaction is the measurement of the successful collaboration among an information system and its learners. It is the range to which learners believe the information system meets their desires. If a system meets the requirements of the users, their satisfaction with the information system will be improved. On the other hand, if the system does not provide essential information, they will become dissatisfied [12]. 


\subsection{Continue to Use}

Continue to use is an indicator used to capture the factors that influence a favorite behavior [13]. The successful of any e-learning system depends on two things, the acceptance of satisfaction and continue to use. Therefore, it was necessary to find out the relevant factors that predict student's continued use of e-learning [14]. Moreover, the intention to use is an essential attributes that defines whether users will really utilize the system or will not really utilizing the system [15]. There are many studies that have found a positive relationship with user's satisfaction with continued usage intention of e-learning systems.

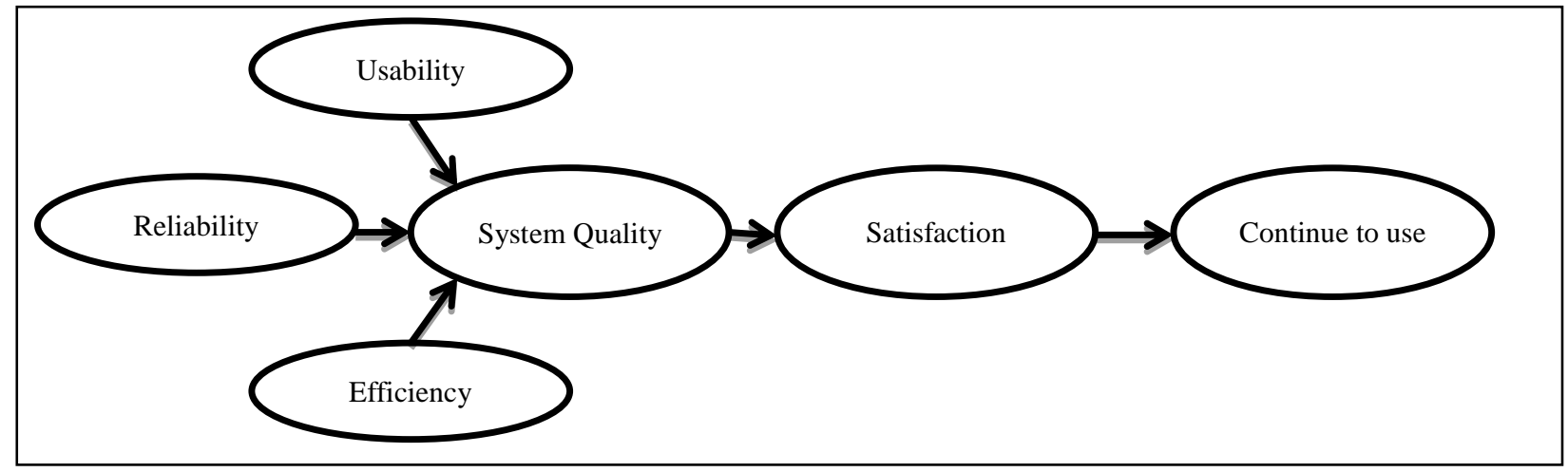

Fig. 1. Proposed framework structure.

\section{Survey Analysis}

\subsection{Data Collection}

Data was collected from 408 students as they were from different public universities in Malaysia, where used a structured questionnaire which was derived from the review of the literature. The questionnaire consisted of 2 sections. The first section was about the demographic information and the second section was about the components of the framework. The demographic information was related to age, gender, and level of study and the experience of using technology. While the components of the framework focused on the usability, reliability, efficiency, system quality, satisfaction and continue to use.

\subsection{Sample Profile}

The demographics of the respondents are the majority of the 236 respondents (57.8\%) were female while 172 respondents (42.2\%) were male. As for the age of the participants, more than three-quarters of the 334 respondents were from 24 years or younger (81.9\%) while 60 respondents (14.7\%) were 25 years to 34 years, and 14 respondents (3.4\%) were from 35 years to 54 years.

As for the education level of the participants, more than half of the 274 respondents $(60.5 \%)$ had bachelor degrees.87 respondents $(21.3 \%)$ were diploma holders while 41 respondents $(10.0 \%)$ were Master's degree holders, and 33 respondents (8.1\%) were Ph.D. degree holders. As for experience with technology, 245 respondents (60\%) had above 3 years, 103 respondents (25.2 \%) had from 1-3 years, while 60 respondents (14.7\%) had less than 1 year.

\subsection{Data Analysis}

In the process of collected data analysis, the structural equation model (SEM) procedure was followed, which was suggested by Anderson and Garbing [16]. This model consists of two major parts that are measurement and structure model. Where was using the measurement model to examine the reliability and discriminant validity which detailed it in Table II, where were all latent variables in the survey had high 
construct reliability. The composite reliability of this study ranging between 0.8776 and 0.9345 . Where, the highest value is $(0.9345)$ for continue to use, while $(0.9325)$ the second value for satisfaction, system quality (0.9168), usability (0.9156), reliability (0.9095), and efficiency (0.8776), and items loading for the 21 items ranged from .745 (SQQ5) to .936 (CQ2). All of the items surpassed the .7 guideline. Similarly, the Cronbach's ranged from 0.7929 to .9034 , with all items surpassing the .7 cutoff. A common measure to establish convergent validity on the construct level is the average variance extracted (AVE). The measurement model's convergent validity is assessed by examining its average variance extracted (AVE) value, which should be higher than (0.5). All constructs have AVE value acceptable which was ranging from 0.7050 to 0.8263 . The highest of AVE value of latent variable is (0.8263) for Continue to use, and the second value for usability (0.7834), satisfaction (0.7755), system quality $(0.6889)$, reliability $(0.7707)$, and efficiency (0.7050).

Table 2. Results of Evaluation the Measurement Model

\begin{tabular}{|c|c|c|c|c|c|}
\hline Latent variable & indicators & Factor Loading & Composite Reliability & AVE & Cronbach's alpha $\alpha$ \\
\hline \multirow{3}{*}{ Usability } & UQI & 0.873 & 0.9156 & 0.7834 & 0.8618 \\
\hline & UQ2 & 0.901 & & & \\
\hline & UQ3 & 0.881 & & & \\
\hline \multirow{3}{*}{ Reliability } & RQ1 & 0.914 & 0.9095 & 0.7707 & 0.8501 \\
\hline & RQ2 & 0.906 & & & \\
\hline & RQ3 & 0.810 & & & \\
\hline \multirow{3}{*}{ Efficiency } & EQ1 & 0.838 & 0.8776 & 0.7050 & 0.7929 \\
\hline & EQ2 & 0.822 & & & \\
\hline & EQ3 & 0.858 & & & \\
\hline \multirow{5}{*}{ System quality } & SQQ1 & 0.849 & 0.9168 & 0.6889 & 0.8857 \\
\hline & SQQ2 & 0.884 & & & \\
\hline & SQQ3 & 0.881 & & & \\
\hline & SQQ4 & 0.783 & & & \\
\hline & SQQ5 & 0.745 & & & \\
\hline \multirow{4}{*}{ Satisfaction } & SQ1 & 0.895 & 0.9325 & 0.7755 & 0.9034 \\
\hline & SQ2 & 0.901 & & & \\
\hline & SQ3 & 0.858 & & & \\
\hline & SQ4 & 0.868 & & & \\
\hline \multirow{3}{*}{ Continue to use } & CQ1 & 0.899 & 0.9345 & 0.8263 & 0.8947 \\
\hline & CQ2 & 0.936 & & & \\
\hline & CQ3 & 0.891 & & & \\
\hline
\end{tabular}

The validity of the structural model is assessed using the coefficient of determination $\left(R^{2}\right)$, path Coefficients, and the strength of the effect $\left(f^{2}\right)$, which demonstrated in Table III. Examining the structural model enables the assessment of its explanatory power. In other words, how much variance in the dependent variables of interest can the independent variable explain or account for.

The $R^{2}$ values move on a quite broad range. Can observe the values between 0.4322 and 0.5770 , i.e. Contingency factors explain the variance of these Latent Variables between $38.40 \%$ and $61.01 \%$. Explaining power is weak at the level of 0.19 , medium at the level of 0.33 , and strong at the level of 0.67 [17].

The usability, efficiency, and reliability can explain around $43.22 \%$ of the variance in system quality, while system quality can explain about $57.70 \%$ of variance in satisfaction, and the last value of satisfaction can explain about $55.34 \%$ of the variance in continue to use The explained all the variances are high a bit of medium. Where, all of the values greater than 0.33 and less than 0.67 . 
The attributes of system quality are usability, reliability and efficiency, which has an impact on system quality as follow; usability small effect at 0.05 , reliability medium effect at 0.16 , and efficiency small effect at 0.04. The main objective of this analysis is the demonstration of the three additional dimensions as a complete measurement model.

Table 3. Results of Evaluation the Structure Models

\begin{tabular}{llllll}
\hline \hline Run & Removed & $\begin{array}{l}\text { R-square } \\
\text { Included }\end{array}$ & $\begin{array}{l}\text { R-square } \\
\text { Excluded }\end{array}$ & Effect Size & Interpretation \\
\hline & Continue to use & 0.5534 & & & \\
& Satisfaction & 0.5770 & & & \\
& System quality & 0.4322 & & & Small effect \\
1 & Usability & & .4002 & 0.05 & Medium effect \\
2 & Reliability & & .3412 & 0.16 & Small effect \\
3 & Efficiency & & .4069 & 0.04 & \\
\hline \hline
\end{tabular}

The result of hypothesizes testing showed significant support for all of hypothesis in this study as shown in Table IV. Where were the attributes of system quality which are usability, reliability, and efficiency are affects directly in system quality it was as follows, usability $(\beta=0.227, t=2.1899, p=0.05)$, reliability $(\beta=0.374$, $t=3.5139, p=0.05)$, and efficiency $(\beta=0.191, t=1.9087, p=0.05)$. As a result, hypotheses $\mathrm{H} 1, \mathrm{H} 2$, and $\mathrm{H} 3$ are supported. Further, by refer to the analysis, the system quality affect directly in satisfaction $(\beta=0.760$, $t=15.4642, p=0.05)$, also satisfaction affect directly in continue to use $(\beta=0.744, t=13.7848, p=0.05)$. As a result, hypotheses $\mathrm{H} 4, \mathrm{H} 5$, are supported.

Table 4. Summary of Hypothesis Testing

\begin{tabular}{lllccccc}
\hline \hline No & \multicolumn{3}{l}{ Hypothesis } & $\beta$ & $t$-value & $p$-value & Support \\
\hline H1 & Usability & $\longrightarrow$ & System quality & 0.227 & 2.1899 & 0.01 & Yes \\
H2 & Reliability & $\longrightarrow$ & System quality & 0.374 & 3.5139 & 0.01 & Yes \\
H3 & Efficiency & $\longrightarrow$ & System quality & 0.191 & 1.9087 & 0.05 & Yes \\
H4 & System quality & $\longrightarrow$ & Satisfaction & 0.760 & 15.4642 & 0.01 & Yes \\
H5 & Satisfaction & $\longrightarrow$ & Continue to use & 0.744 & 13.7848 & 0.01 & Yes \\
\hline \hline
\end{tabular}

\section{Discussion and Conclusion}

The purpose of this study was to test the top three attributes of system quality in a public university in Malaysia. The study also examined the relationships between system quality and user satisfaction, and the impact of user satisfaction on usage continuance. The model adopted in this study explain that usability, efficiency, and reliability were positively related to system quality explaining the around $43.22 \%$ of the variance in system quality, while system quality was positively related to satisfaction explaining about $57.70 \%$ of variance in satisfaction, and the last value of satisfaction was positively related to continuing to use explaining about $55.34 \%$ of the variance in intention to use. In sum-up, this study involved the system quality factors, specifically within the end-users' factors, which found that usability, reliability, and efficiency affected the system quality. Moreover, system quality is a significant attribute influencing user satisfaction in using an e-learning system. User satisfaction was also found to be significant in affecting users' intention to use. The obtained results of this study will assist developers to develop e-learning systems by adopting these factors which finally affect the user satisfaction in the aspect of continue to use. Where was presented by the study may give reasons for the developers to improve approaches to enhance e-learning system quality by focusing on these attributes usability, reliability and efficiency combined. In the future work, more efforts should be applied for more research to improve the overall study and to addresses its limitations because this study shed light on the side of the end users. As for the 
recommendations of this study, supposed to recommend the focusing on other quality elements such as the information quality, the service quality. Furthermore, it should concentrate on the other recipients of the e-learning system. Finally, the fulfilled result of this study wished to give a general awareness and understanding on the user satisfaction and continue to use in order to maximize the actual use of e-learning systems in general.

\section{Acknowledgment}

This research paper is made possible through the help and support from everyone. Especially, I dedicate my acknowledgment for my supervisors' gratitude toward the counseling and contribute: First and foremost, I would like to show my great gratitude to Dr. Nurlida Basir for her most support and encouragement. She kindly read this paper and offered valuable detailed advices on grammar, organization, and the theme of the paper which made the possibility of publishing this paper. Second, I would like also to express my deep appreciation to Dr. Norasikin Binti Fabil for her reading my paper and providing valuable advices on the content of this paper. Finally, the completion of this paper could not have possible without the kind support and help of my parents.

\section{References}

[1] Alkhattabi, M., Neagu, D., \& Cullen, A. (2010). Information quality framework for e-learning systems. Knowledge Management and e-Learning: An International Journal (KM\&EL), 2(4), 340-362.

[2] Lee, M. C. (2010). Explaining and predicting users' continuance intention toward e-learning: An extension of the expectation-confirmation model. Computers and Education, 54(2), 506-516.

[3] Zanjani, M., Vali, F., \& Ramazani, M. (2012). Investigation of e-learning acceptance in teaching English language based on TAM model. ARPN Journal of Systems and Software, 2(11).

[4] Rostaminezhad, M. A., Mozayani, N., Norozi, D., \& Iziy, M. (2013). Factors related to e-learner dropout: Case study of IUST elearning center. Procedia-Social and Behavioral Sciences, 83, 522-527.

[5] Al-Qutaish, R. E. (2010). Quality models in software engineering literature: An analytical and comparative study. Journal of American Science, 6(3), 166-175.

[6] Dubey, S. K., Ghosh, S., \& Rana, A. (2012). Comparison of software quality models: An analytical approach. International Journal of Emerging Technology and Advanced Engineering, 2(2), 111-119.

[7] Delone, W. H., \& McLean, E. R. (2003). The Delone and McLean model of information systems success: A ten-year update. Journal of Management Information Systems, 19(4), 9-30.

[8] Madan, A., \& Dubey, S. K. (2012). Usability evaluation methods: A literature review. International Journal of Engineering Science and Technology, 4(2).

[9] Ardito, C., Costabile, M. F., Marsico, M., Lanzilotti, R., Levialdi, S., Roselli, T., \& Rossano, V. (2006). An approach to usability evaluation of e-learning applications. Universal Access in the Information Society, 4(3), 270-283.

[10] Osaki, S. (2012). Stochastic models in reliability and maintenance. Springer Science \& Business Media (p. 253). Berlin: Springer.

[11] Papanikolaou, K., \& Mavromoustakos, S. (2008). Web2train: A design model for corporate e-learning systems. BIS (Workshops) (pp. 155-163).

[12] Freeze, R. D., Alshare, K. A., Lane, P. L., \& Joseph Wen, H. (2010). IS success model in e-learning context based on students' perceptions. Journal of Information Systems Education, 21(2), 173.

[13] Punnoose, A. (2012). Determinants of intention to use e-learning based on the technology acceptance model. Journal of Information Technology Education: Research, 11(1), 301-337.

[14] Lwoga, E. T. (2014). Critical success factors for adoption of web-based learning management systems in 
Tanzania. International Journal of Education \& Development Using Information \& Communication Technology, 10(1), 4-21.

[15] Shroff, R. H., Deneen, C. D., \& Ng, E. M. (2011). Analysis of the technology acceptance model in examining students' behavioural intention to use an e-portfolio system. Australasian Journal of Educational Technology, 27(4), 600-618.

[16] Anderson, J. C., \& Gerbing, D. W. (1988). Structural equation modeling in practice: A review and recommended two-step approach. Psychological Bulletin, 103 (3), 411-423.

[17] Chin, W. W. (1998). The partial least squares approach to structural equation modeling. Modern Methods for Business Research, 295(2), 295-336.

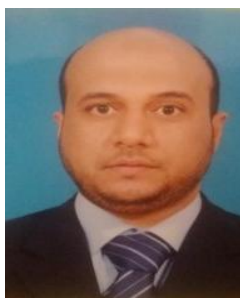

Abdulhakim Elmoawe Dreheeb was born in 1976 Tripoli-Libya. He received his BSc in systems programming and analysis (2004) at Ibn Elheithem Higher Center for Technological Education and Scientific Research, Tripoli, Libya. He earned his MSc in information technology in (2007) at University Utara Malaysia, Kedah, Malaysia. He is a fourth-year student of a doctor of philosophy degree at University Science Islam Malaysia, Nilai, Malaysia. His interests are primarily on system applications on e-learning, quality of e-learning systems.

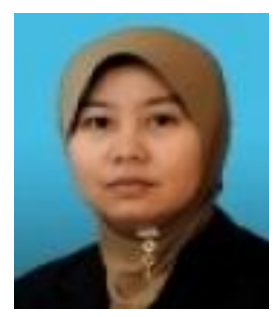

Nurlida Basir is a doctor at the Faculty of Science and Technology in University Science Islam Malaysia. Her area of specialization and research interests are the software engineering.

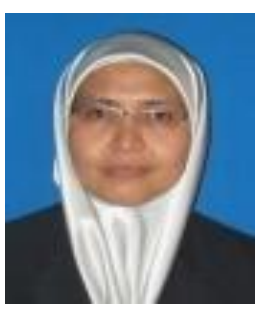

Norasikin Binti fabil is a doctor at the Faculty of Science and Technology in University Science Islam Malaysia. Her areas of specialization and research interests are the computer graphic and animation (Multimedia) as well as computer assisted instruction (CAI). 